\title{
Artikel
}

Michaela Ott*

\section{Vom Schwarz-Weiß-Kontrast zur dividuellen Kompositkultur}


Abstract: I discuss current shifts in cultural understandings under postcolonial conditions with particular regard to the French-African-Antillean area. Through a short reconstruction of culture constitutive approaches, their continuation and criticism in the Antillean area and furthermore the Afropolitan interpretations by Mbembe,Enwezor and African artists, I come to the conclusion that we need an epistemological shift in the cultural studies discourse itself. Along the lines of the affirmative-critical aesthetic of the mentioned African theorists, curators and artists, I advocate that the cultural studies discourse distances itself from descriptions in terms of cultural contrast, of the same and the other, of white and black and so on. I argue that the discourse should abandon the idea of unified or oppositional cultures and instead emphasize the "composite-cultural", i.e. the entanglements of respective personal or societal forms of articulation and existence as well as profile the types of symbolic interpenetration, the temporally and aesthetically conditioned "dividuation". With examples from the African art context, I attempt to outline certain dividual procedures and to stress the fact that nowadays even western articulations are bound to endure forced cultural participation [Zwangsteilhabe]: Instead of discursive contrasts we are in need of analyses of the respective participation and (self-)dividuation processes.

Keywords: Alterität, Kompositkulturen, post-ethnische Identitäten, Afropolitanismus, Teilhabe, Dividuation.

*Prof. Michaela Ott, Hochschule für Bildende Künste, Hamburg, GERMANY, email: philott@arcor.de

\section{Von dualistischen zu dividuellen Kulturkonzepten}

Lauscht man (post)kolonialen Theoretiker*innen der Gegenwart mit afrikanischem oder asiatischem Hintergrund, so bestimmen sie ihre Sprecher*innenposition heute zumeist als eine aus dem globalen Süden. Auch Filmemacher*innen in Tunis sprechen vom Süden, um ihr symbolisches Zuhause zu verorten und sich von der westlich/ nördlichen Welt zu distanzieren. Die Abgrenzung eines globalen Südens vom Norden ist seit geraumer Zeit an die Stelle der Oppositionspaare von Okzident/Orient resp. Weißer und Schwarzer ${ }^{1}$ Welt getreten, welche symbolische Ungleichgewichte und kulturelle Bevormundung anmahnten. Als geopolitisch-ökonomischer Begriff will das Süd-Nord-Paar vor allem wirtschaftliche Asymmetrien und Hierarchien in Sachen Fortschritt und technischer Entwicklung signalisieren.

1 Im Sinne der Markierung der behelfsmäßigen, künstlichen und asymmetrischen begrifflichen Unterscheidung werden ,Schwarz' und ,Weiß' durchgängig großgeschrieben.
Mittlerweile mehren sich allerdings die Stimmen, die jede dualistische Entgegensetzung und jede Bestimmung von „Andersheit" für nicht länger erkenntnisträchtig erklären, da die gegenwärtige Welt durch verstärkte kulturelle Einflussnahmen und derart tiefgreifende politisch-wirtschaftliche Interdependenzen gekennzeichnet sei, dass die einzelne Situation besser über ihre je spezifische Einbindung in die weltumspannenden Austauschprozesse und ihre besondere Art der politisch-kulturellen Teilhabe zu charakterisieren sei. "Verflechtung" ist der Terminus, der heute bevorzugt zur Wiedergabe des globalisierungsbedingten Ineinanders des wirtschaftlichen und kulturellen Geschehens herangezogen wird - und uns dazu auffordert, die Spezifik der jeweiligen Teilhabe in entsprechenden Begriffen und Beschreibungsweisen zu analysieren.

So treten an die Stelle vormaliger "Globalgeschichten", welchen eine unerwünschte Homogenisierung der Weltenvielfalt unter westlicher Logik vorgeworfen wird, in Nähe zu den Begriffen der "Cultures composites" von Edouard Glissant² oder

2 Edouard Glissant, Traité du Tout-Monde, Paris: Gallimard, 1997, 195. 
des "Afropolitanen" von Achille Mbembe $^{3}$ vermehrt "entangled histories". Diese auf je besondere Weltausschnitte fokussierenden Geschichten berichten, in Vermeidung einer chronologisierten Entwicklungslogik, von Koexistenzen und Interferenzen verschiedenkultureller Schichten und Artikulationsweisen, von wechselseitigen Bedingtheiten von Alt und Neu, Nah und Fern und konturieren vielstimmige, unter Umständen disharmonische Komposite. Aus ihrem Blickwinkel erscheint die Entgegensetzung von Eigenem und Anderem, von Zentrum und Peripherie schon aufgrund der darin mitschwingenden Bewertungen obsolet. Andererseits machen gerade sie deutlich, dass auf Angaben zu je partikularer "Andersheit" nicht verzichtet werden kann, da ein Hinhören auf untergründig Mitsprechende überhaupt erst Personen, Stimmen, kulturelle Zusammenhänge hervortreten lässt, die im nördlichen Diskurs bis dato kaum wahrgenommen worden sind. Im Extremfall verweist die Beachtung kultureller Ausschlüsse auf Fälle absoluter Alterität, deren Differenz, einer anderen als der westlich/nördlichen Logik und Ethik gehorchend, nicht vermittelbar, nicht in westliche Sprachen und in überhaupt keine Sprache übersetzbar erscheint. ${ }^{4}$

Der Verweis auf Andersheit war aber lange schon und ist weiterhin auch vonnöten, um über wissenschaftliche Disziplingrenzen hinaus zu reflektieren und miteinzuschließen, was bis dato durch epistemische Raster gefallen und außen vor geblieben ist. Theoretische Inklusionsbestrebungen, die disziplintransversal operieren, betonen dann unter Umständen, dass als "individuell" verstandene Personen, Kulturen und Kunstwerke so ungeteilt nicht sind, sondern mit qualitativ verschiedenen "Anderen" konstitutive Teilhabeverhältnisse eingehen, welche erst ermöglichen, dass es sie in dieser spannungsreichen Komplexität gibt. Auch die Weiße Welt erkennt sich heute in diesem Sinn als vielfarbig, sucht ihre behauptete Weißheit zu durchleuchten und sich von unhinterfragten Selbstverständnissen zu distanzieren.

3 Achille Mbembe, Ausgang aus der langen Nacht. Versuch über ein entkolonisiertes Afrika, Berlin: Suhrkamp, 2016, 275-295.

4 Vgl. Gayatri Chakravorty Spivak, A Critique of Postcolonial Reason. Toward a History of the Vanishing Past, Cambridge (Mass)/London: Harvard Univ. Press, 1999.
Blinde Flecken werden seit den 1990er Jahren aber auch in jenen Positionen, die sich über eine Abstandnahme vom westlich/nördlichen Diskurs definieren, angemahnt. So wirft der afroenglische Kulturwissenschaftler Paul Gilroy in seiner einflussreichen Schrift The Black Atlantic (1993) ${ }^{5}$ just dem Schwarzen (und karibischen) Diskurs vor, auf der Suche nach Authentizität seine Verflechtung mit den Weißen Diskursen zu übersehen und deren Bedeutung für die eigene Konstitution zu verkennen. Nicht nur nähmen Weiße Theoretiker*innen in der Regel Schwarze Diskurse nicht wahr; auch Schwarze leugneten gelegentlich den Tribut, den sie Weißen symbolischen Setzungen schulden. Im Dienste einer Vorstellung von homogener Kultur würden sie die kulturellen und begrifflichen Ko-Konstitutionen nicht fruchtbar machen. Ethnische Gegensatzbildungen und diskursive Abschottungen, so Gilroys Befund, kennzeichneten beide Seiten.

In Zeiten verstärkter informationeller, ästhetischer, personaler und wirtschaftlicher Verflechtung spitzt sich das Beschreibungsproblem klarerweise zu; kulturelle Verfasstheiten, die hier insbesondere interessieren, lassen sich weniger denn je in dualistischen Begriffspaaren fassen. Wie Stuart Hall als einer der ersten (post)kolonialer Theoretiker betont, bringt die Migration von Personen und Kulturen zwangsläufig vieldirektionale Orientierungen und Identitätsfragmentierungen oder besser-multiplikationen mit sich, die die nationalstaatlich oder territorial bestimmten Ankunftskulturen transformieren und ihrerseits enthomogenisieren. ${ }^{6}$ Was allerdings für zahlreiche Länder des Nordens und Südens seit Langem Normalität ist, die Einwanderung von Bevölkerungen mit unterschiedlichen Sprachen, Kulturen und Religionen und die dadurch potenzierte kulturelle Durchmischung der Gesellschaft, wird in Deutschland und den osteuropäischen Ländern erst heute als Herausforderung wahrgenommen, der sie sich nur widerwillig, wenn überhaupt, zu stellen versuchen.

5 Paul Gilroy, The Black Atlantic. Modernity and Double Consciousness, London/New York: Verso, 1993.

6 Stuart Hall, Wann gab es, das Postkoloniale'? Denken an der Grenze. In: Sebastian Conrad et al. (Hgg.), Jenseits des Eurozentrismus. Postkoloniale Perspektiven in den Geschichts- und Kulturwissenschaften, Frankfurt/M./New York: Campus Verlag, 2002, 197-223 (212). 
Kulturelle (Inter)Dependenzen zu erörtern und im besten Fall fruchtbar zu machen, ist heute das Anliegen zahlreicher Theoretiker*innen und Künstler*innen mit asiatischem oder afrikanischem Hintergrund. Der (post)koloniale Diskurs wurde nicht zufällig von Wissenschaftler*innen, die zwischen verschiedenen Kulturen leben und arbeiten, zwischen Europa, der Karibik, dem Nahen Osten oder Afrika, zwischen den USA, Indien oder China in die Welt gebracht. Seit Jahrzehnten kritisiert er die Einseitigkeit und Voreingenommenheit westlich/nördlicher, damit als provinziell gekennzeichneter Perspektiven und methodischer Zugriffe, ihr Übersehen mitenthaltener kulturdifferenter "Anderer"; er wendet sich gegen die universalisierende Einebnung von Differenz wie gegen empirieferne Fetischisierung von Alterität, auch die epistemologische Suche nach Heterotopien, wo die landeseigene Heterotopie, die Nicht-Teilhabe von Anderskulturellen an gesellschaftlicher Partizipation, nicht ins Blickfeld rückt.

Wie Achille Mbembe neuerdings verstärkt anmahnt, ist das kulturelle Geschehen, auch die Frage der Restitution afrikanischer Kunstgegenstände und Kultobjekte, nicht länger im Dualismus von Hier und Dort, sondern der Teilhabe oder, wie ich sagen möchte, der Dividuation ${ }^{7}$ zu konzipieren. Diese Termini zielen auf Vorstellungen und kulturpolitische Handlungsweisen, die das Interferierende und partiell Ineinandergreifende, eher Schnittmengen als Sonderstellungen anvisieren. Dividuation erscheint als Begriff besonders geeignet, da er, auf die philosophischen und anthropologischen Begriffe des "Dividuums" und des „Dividuellen" zurückgehend, unterschiedliche Prozesse der (Selbst)unter- und -aufteilung, der Teilhabe an qualitativ verschiedenen "Anderen" wie der Vereinnahmung durch "Anderes" akzentuiert. Um nicht-eindeutige Grenzen zwischen Person und Gesellschaft wiederzugeben, wählt die englische Anthropologin Marilyn Strathern ${ }^{8}$ den Begriff des "Dividuellen" in den ausgehenden 1980er Jahren; sie hebt damit die Institutionalisierung nicht-dualistischer Verhältnisse zwischen dem Einzelnem und der Gesellschaft in melanesischen Geschenkökonomien hervor. Der französische Philosoph

7 Vgl. Michaela Ott, Dividuationen. Theorien der Teilhabe, Berlin: b_books, 2015.

8 Marilyn Strathern, The Gender of Gift, Los Angeles: Univ. of California Press, 1988, 13.
Gilles Deleuze bringt etwa zeitgleich das Adjektiv "dividuell" zur Charakterisierung von filmischen und musikalischen Kunstwerken in Einsatz, die durch zeitbedingte Transformationen und eine konzeptuelle Nichtfestlegbarkeit ausgewiesen sind. ${ }^{9}$ Mitte der 1990 er Jahre charakterisiert er allerdings mit negativem Vorzeichen die technologiebedingten "Verflüssigungen" menschlicher Subjektivierungsprozesse als "dividuell"10. In Verlängerung und Zuspitzung dieser begrifflichen Vorgaben möchte ich den Begriff der Dividuation hier zur Bestimmung zeitgenössischer Kompositkulturen verwenden, die durch verschiedene Prozesse der Überlappung und Amalgamierung wie durch harte Unterscheidungen und Abgrenzungen gekennzeichnet sind. Der Begriff der Dividuation soll zur Durchleuchtung vermeintlich ungeteilter kultureller Gefüge auf die mitenthaltenen "Anderen" hin dienen, aber auch die Grenzen der Teilhabe, Vorgänge der Auf- und Unterteilung markieren. Kulturelle Artikulationen formen sich heute schon aufgrund der digitalen Appropriierungsmöglichkeiten und der kommunizierten Hypes zu ästhetisch-medialen Mischgebilden aus, die ihre Aussagekraft häufig gerade aus der Komposition von Kulturdifferentem beziehen. Edouard Glissants bis heute wenig beachteter Terminus, der die verschiedenen Zusammensetzungen von Kompositen bis hin zu immanenten, nicht-aufklärbaren Differenzen betont, scheint mir daher besser als jener des biologischen „Hybrids" oder der "intersecting cultures" geeignet, ihre vieldeutige Problematik, auch das Kontingente der dividuellen Komposition hervorzukehren.

Dass sich heute trotz des losen kulturellen Zusammenhangs von indigen genannten Gruppen gleichwohl spezifische Differenzen ausmachen lassen, stimmt einen Anthropologen wie James Clifford hoffnungsfroh. Sei es doch erstaunlich, so sein jüngstes Statement ${ }^{11}$, dass die indigenen Gruppierungen trotz der vielfältigen Anpassungszwänge an umgebende Kulturen überleben und zum Teil sogar wiedererstarken, wenn auch nur

9 Gilles Deleuze, Das Bewegungs-Bild, Kino 1, Frankfurt/M.: Suhrkamp, 1997, 129.

10 Gilles Deleuze, Postscriptum zur Kontrollgesellschaft. In: Unterhandlungen, Frankfurt/M.: Suhrkamp, 1993, 254-262.

11 Abgegeben bei der Konferenz "Neolithische Kindheit" im HKW Berlin, 26.05.2018. 
in fortgesetzt neu auszutarierender Verbindung, in einer zugespitzten Form der Dividuation.

Derartig losen Verbindungen stehen heute bekanntlich harte kulturelle Abgrenzungen und Rassismen gegenüber, die in der Gegenwart ein immer weniger umstrittenes Comeback feiern, das mit unverhohlenen Abwertungen anderskultureller und -religiöser Traditionen einhergehen kann. Im Sinne der Errettung spezifischer Kulturen, Religionen und politischer Systeme halten nationalkonservative Strömungen dafür, zwischen dem als eigen und als fremd Betrachteten erneut hart zu unterscheiden und sich vehement gegen ein Außen zu wehren, das die kulturelle Besonderheit zu "unterwandern" tendiert. Im heutigen Europa wird visuellen Personenmerkmalen wieder politische Aufmerksamkeit geschenkt und in kulturellen Begegnungen das mitenthaltene "Gegen" hervorgekehrt.

In theoretischer Zuspitzung dieser Entwicklungstendenzen wenden sich Imperialismustheoretiker*innen wie Laura Stolter ${ }^{12}$ mit Vehemenz gegen eine Beschreibung der Globalisierung als zunehmenden Vermischungs- und Egalisierungsprozess, da sie in ihr nur eine beschönigende Wiedergabe weiterbestehender und längst nicht überwundener Herrschaftsverhältnisse erblicken. Stolter wirft den Cultural Studies und ihrer Fokussierung auf kulturelle Hybridisierungen vor, die immer noch andauernden Kolonialverhältnisse zu übersehen, welche die Welt hart in Nord und Süd unterteilen und Ausbeutungsstrategien und wirtschaftliche Ungleichteilhaben perennieren.

Aufgrund der skizzierten markanten Unterschiede in Sachen Gegenwartsdiagnose und Gewichtung kultureller Prozesse findet der kulturwissenschaftliche Diskurs der Gegenwart zu der salomonischen Formel, dass je nach perspektivischer Rahmung, nach Erkenntnisinteresse und gewähltem Vokabular sich die kulturelle Lage bald so, bald anders beschreiben und bewerten lässt. Unstrittig scheint zu sein, dass sich trotz Transnationalisierung diskursiver, ästhetischer und kultureller Gehalte oder gerade deswegen eine neue Inwertsetzung von Identität und kultureller Zugehörigkeit beobachten lässt. Diese wird immer mehr als kostbares Gut gehandelt,

$12 \mathrm{Vgl}$. Ann Laura Stoler, Race and the Education of Desire: Foucault's 'History of Sexuality' and the Colonial Order of Things, Durham/London: Duke University Press, 1995. freilich unterschiedlich komposit- oder monokulturell bestimmt und von "Anderem" abgesetzt. Im letzteren Fall wird sie mit der Aufforderung verbunden, wohlweislich zu bedenken, an wen sie vergeben, wer inkludiert werden darf und wer in einem Außerhalb dazu verbleiben muss. Ein neuer eurozentrischer und allgemein nationalkultureller Geiz macht sich breit.

Dabei ist die Geschichte der europäischen Identitätskonstruktion seit jeher durch unscharfe Bestimmungen des Eigenen und Anderen charakterisiert. Europa ist ein willkürlich abgegrenztes, mythisch begründetes Konstrukt, das von Frauenraub und Übergriffen zwischen europäischen und asiatischen Regionen in literarischen Texten wie der Ilias und im Medea-Mythos erzählt. Die afrikanische Geschichtsschreibung des Senegalesen Cheikh Anta Diop legt daher nahe, die europäische Geschichte über diese willkürlich gezogenen Grenzen hinaus zu denken und als von schwarzafrikanischen Ägyptern mit auf den Weg gebracht zu erkennen. Heute ließe sich vor allem vorbringen, dass Europa nicht an seinen erneut markierten und bewehrten Außengrenzen endet, unterhalten doch Länder wie Frankreich, Großbritannien, Spanien und Portugal nach wie vor kulturelle Weltreiche, wenn nur die Verbreitung und Dominanz ihrer Sprachen und Kulturen in Rechnung gestellt wird. Andere europäische Länder wie etwa Deutschland suchen ihrerseits weltweit ökonomisch und kulturell zu interagieren und ihren Einflussbereich zu erweitern.

Aus diesem Grund scheint es angeraten, sich noch einmal von Theoretiker*innen unterschiedlicher nicht-europäischer Herkunft über ihren Umgang mit kulturellen Differenzen und deren prekärer Vereinbarkeit belehren zu lassen. Im Hinblick darauf lohnt ein erneuter Blick auf vergangene Aushandlungsprozesse zwischen Schwarzen und Weißen Diskursen, um sodann deren zeitgenössische (post)koloniale Weiterführung, deren Versuche der Verminderung des hautfarbenbezogenen Kontrasts in prozessbetonenden Theorien mit der genannten Begrifflichkeit der Dividuation zu unterstützen, welche, wie skizziert, das Ineinander der Welten, aber auch die spezifischen Reidentifizierungen der kulturellen Komposite und inr Verhältnis zu sich verschiebenden Herrschaftskomplexen erfassen will.

Ein möglicher Weg des Nachvollzugs der diskursiv-kulturellen Aushandlungsprozesse ver- 
läuft von Europa über Afrika in die Antillen, wo die kompositkulturellen Existenzweisen und ihre ästhetischen Varianten bereits vor geraumen Jahrzehnten theoretisiert worden sind. Im europäisch-afrikanisch-antillanischen und vorzugsweise französischsprachigen Raum fällt dabei auf, dass der Begriff von Kultur nicht nur zwischen den Kontinenten hin- und hergeschoben, sondern fortgesetzt kritisiert und durch adäquatere Bezeichnungen im Sinne der Inkludierung "anderer" Artikulationen ersetzt worden ist. Unüberhörbar teilt er mit, dass Identität heute nur als Kompromiss auf der Basis multipler und schwer zu verfugender Orientierungen verstanden werden kann und dass die temporäre Abstimmung und Eingemeindung von Verschiedenkulturellem prekäre psychische Ausbalancierungen erforderlich macht. Er weist auch darauf hin, dass sich nicht nur personale Einzelne, sondern auch Kunstwerke und Kulturen sukzessive ihrer Individualität, ihrer Ungeteiltheit, begeben und daher neue Begriffe an ihre Stelle zu setzen sind.

\section{Deutungen von Schwarz zwischen Kultur und Ökonomie}

Die Geschichte der kulturellen Begegnung zwischen Europa und Afrika, deren bessere Wiederholung heute von dem kamerunischen Filmemacher Jean-Pierre Bekolo eingefordert wird, erfolgt zunächst unidirektional von Europa aus: Im Sinne der Aneignung des kulturell fremden "Anderen" nehmen mit dem offiziellen Beginn der europäischen Kolonisierung des afrikanischen Kontinents ab 1884 die Deutungen dieses "Anderen" zu. Zum Zweck der anthropologischen Unterscheidung von Rassen, Kulturen, Denkmustern und Affekthaltungen werden krude Zuschreibungen durch Weiße Diskurse getätigt, die von aus Afrika stammenden Theoretikern teilweise aufgegriffen, gegenbesetzt, umgekehrt, modifiziert und verallgemeinert werden. Die "Kultur Afrikas" ist ein sprechendes Beispiel für begriffliche Übertragungen und semantische Verschiebungen, eine Bestätigung der These, dass Kultur aus - auch missverständlichen - Übersetzungsvorgängen entsteht. ${ }^{13}$

13 Doris Bachmann-Medick im Gespräch mit Boris Buden, in: Boris Buden/Stefan Nowotny (Hgg.), Übersetzung:
Der französische Philosoph Lucien LévyBruhl, ${ }^{14}$ der ab den 1910er Jahren die universalisierenden Annahmen der Kant'schen Philosophie bezüglich der Struktur menschlicher Erkenntnisvermögen in Frage zu stellen sucht, führt gegen deren vermeinte Allgemeingültigkeit eine „primitive Mentalität" ins Diskursfeld ein. Dieser Mentalität glaubt er eine "Prälogik" attestieren zu können, welche nichts vom Kausalitätsprinzip wisse, dafür Arten magischer Partizipation mit anderen Raum-, Zeit- und Wirkungswahrnehmungen kenne. In die Reihe seiner Kritiker reiht sich später Claude Lévi-Strauss mit seiner Schrift Das wilde Denken von 1962 ein. ${ }^{15}$ Dieser betont mit Vehemenz, dass magische Denkweisen nicht kategorial von wissenschaftlichen zu unterschieden seien, vielmehr von unterschiedlichen Logiken auf verschiedenen strategischen Ebenen auszugehen sei. Schließlich wiesen auch Mythen und Rituale Beobachtungs- und Reflexionsmodi auf, allerdings angepasst an die Erforschung der sinnlich konkreten Welt. Zudem operiere das "wilde" bzw. "primitive" Denken nicht mittels Partizipation, sondern mittels Unterscheidung und Gegensatzbildung wie andere Wissenschaften auch.

Und doch lebt Lévy-Bruhls Unterscheidung zweier Denkweisen, wenn auch polemisch modifiziert, bis heute weiter, wie die jüngste Schrift von Achille Mbembe schon in ihrem Titel Critique de la raison nègre ${ }^{16}$ von 2013 verrät - ein Titel, dessen polemische Umwertung in der deutschen Übersetzung zur Kritik der Schwarzen Vernunft abgeschwächt worden ist. Das der, raison nègre' vorangehende und von Mbembe umgedeutete Konzept der "Négritude", welches der senegalesische Dichter und Staatsmann Leopold Sédar Senghor ab den 1930er Jahren - zusammen mit karibischen Autoren wie Aimé Césaire in Paris unter dem Eindruck der dort musealisierten "afrikanischen" Kunstwerke - entwickelt, gründet seinerseits auf der Annahme kulturdifferen-

Das Versprechen eines Begriffs, Wien: Turia + Kant, 2008, 29-42 (34).

$14 \mathrm{Vgl}$. Lucien Lévy-Bruhl, Les fonctions mentales dans les sociétés inférieures, Paris: Alcan, 1910; La mentalité primitive, Paris: Presses univ. de France, 1922.

15 Claude Lévi-Strauss, La pensée sauvage, Paris: Plon, 1962, 28-30.

16 Achille Mbembe, Critique de la raison nègre, Paris: La Découverte, 2013; Kritik der Schwarzen Vernunft, Berlin: Suhrkamp, 2014. 
ter menschlicher Vermögen: So schreibt Senghor den Hellenen die Vernunft, den Schwarzen dagegen eine höherwertige Emotionalität zu. Nur Letztere bringe eine lebendige "con-naissance" hervor, eine Erkenntnis als "Mitgeburt", aus welcher sich das Wesen der "Négritude", ihrer Kunst und ihrer Neigung zu Musik, Rhythmus und Tanz ableiten lasse. ${ }^{17}$ Das "Prälogische" wird hier in ausgeprägte Emotionalität weiterübersetzt. Später $^{18}$ dagegen lässt Senghor - unter dem Eindruck einer Bergson-Lektüre - diese Entgegensetzung fallen und eine vereinheitlichte Vermögenskonzeption an ihre Stelle treten, eine „Berührungsvernunft", dank welcher den Schwarzen nun Intuition und Vernunft gleichermaßen zugesprochen wird. Senghors Denken erweist sich selbst als sympathetische Vernunft, insofern er sich von Bergsons Symbiose von Intuition und Rationalität ${ }^{19}$ anstecken lässt und sich diese Position aneignet, um kurioserweise aus ihr ein neues afrikanisches Selbstbewusstsein zu beziehen.

In das von inm gesuchte panafrikanische Kulturverständnis werden auch deutsche Begriffe und Deutungen integriert. So wollen Senghor und Césaire just von Leo Frobenius, dem ersten Deutschen, der sich systematisch mit Kunstwerken auf dem afrikanischen Kontinent befasst, ein Verständnis von afrikanischer Kultur als geschichtlicher und mystischer Überlieferung übernommen haben, ${ }^{20}$ obwohl sie dessen Schrift Kulturgeschichte Afrikas ${ }^{21}$ zunächst in der französischen Übersetzung als Histoire de la civilisation [!] africaine (1936) rezipieren. Frobenius wiederum will in den altafrikanischen Felsritzungen und -zeichnungen überall einen "gleichen Geist, einen gleichen Charakter und eine gleiche Natur"22 entdecken. Und doch untergräbt er selbst seine Behauptung kultureller Gleichheit und Konti-

17 Leopold Sédar Senghor. Négritude und Humanismus, Düsseldorf: Diederichs, 1967.

$18 \mathrm{Vgl}$. Souleymane Bachir Diagne, Bergson postcolonial, Paris: CNRS Ed., 2010.

19 Henri Bergson, Essai sur les données immédiates de la conscience. Paris: Presses univ. de France, 1889.

20 Léopold Sédar Senghor, Négritude et Germanisme. Tübingen/Basel: Horst Erdmann, 1968, 11.

21 Leo Frobenius. Kulturgeschichte Afrikas. Prolegomena zu einer historischen Gestaltlehre. Frankfurt/M.: Phaidon, 1933.

22 Leo Frobenius. Kulturgeschichte Afrikas. Prolegomena zu einer historischen Gestaltlehre. Frankfurt/M.: Phaidon, 1933, 15. nuität, indem er sie auf der Basis künstlerischer Zeugnisse zu einer "Zwei-Kulturen-Theorie"23 erweitert und die "äthiopische" Gemütskultur zu einer "hamitischen" Vernunftkultur in Gegensatz bringt. Senghor integriert diese Zwei-Kulturen-Theorie wiederum in sein Konzept panafrikanischer Gesamtidentität: Er gemeindet beide als komplementäre, eher geographisch-kulturell denn rassisch unterschiedene, in die gewünschte "Africanité" ein. Seine und Frobenius' dualistische Unterteilung Afrikas lebt gleichwohl bis heute weiter, insofern nach wie vor zwischen dem südsaharischen und arabischen Kulturraum unterschieden und nur Ersterer für wirklich afrikanisch erachtet wird.

Achille Mbembe lässt Senghors Unterscheidung von Emotionalität und Vernunft noch einmal aufleben, wenn er die den Schwarzen zugeschriebene Nähe zum Ursprünglichen, Emotionalen und Animalischen sarkastisch kommentiert. Und doch sucht er diese Dualität umgehend in einem allgemeinen Vermögen "Schwarzer Vernunft", einem general Black intellect, aufzuheben, welchen er nicht nur Afrikaner*innen, sondern all jenen „Verdammten dieser Erde" zuerkennen will, die heute zur „überflüssigen Menschheit"24 gezählt werden und daher "erneut nicht ganz Menschen sind". Auf die Erkenntnis, dass kulturelle Entgegensetzungen in affektgeladenen und gewaltstiftenden Akten münden, antwortet er mit der Erweiterung der "Négritude"-Konzeption zu einer globalisierten, polemisch Schwarz genannten Vernunft, in deren Vorzug man nicht nach Maßgabe kulturell-rassistischer Unterteilungen, sondern ökonomisch-symbolischer (Nicht-)Teilhaben gelangt. Wenn Mbembe unter die "conditio nigra" alle von den Wertschöpfungsketten Ausgeschlossenen subsumiert, tritt die Frage nach kultureller Differenz in den Hintergrund. Das Kompositkulturelle erscheint dann gesetzt: Die hier Adressierten, häufig Arbeitsmigrant*innen aus dem globalen Süden, welche unterschiedliche Identitätsangebote in sich vereinen müssen, finden sich zwangsläufig zu diasporischen Gemeinden und prekären kulturellen Ensembles zusammen. Eine mini-

23 Leo Frobenius. Kulturgeschichte Afrikas. Prolegomena zu einer historischen Gestaltlehre. Frankfurt/M.: Phaidon, 1933, 234-241.

24 Achille Mbembe, Kritik der Schwarzen Vernunft, Berlin: Suhrkamp, 2015, 16. 
male Identität erwächst ihnen dann aus einem "piecing together more-than-local patterns", wie James Clifford diese Art der Kulturkomposition nennt. ${ }^{25}$ Diese konsolidiert sich weniger über historische Rückgriffe oder futuristische Antizipationen als über "looping lines of recollection, and specific paths forward". ${ }^{26}$ Befreiung von Tradition und Vergangenheit und disparate Kombinationen räumlicher und zeitlicher Muster scheinen gerade die Voraussetzung für eine Einfügung in fremde Kulturzusammenhänge zu sein. Zeitgestückelte Verortung ergebe sich dann aus ad hoc gewählten, (nicht-in)dividuellen Teilhaben, ohne Synthetisierung zu einer klar umrissenen Identität.

\section{Kompositkulturelle Bestimmungen}

Interessanterweise benennt Sartre schon 1948, in seinem Vorwort zur von Senghor herausgegebenen Anthologie de la Nouvelle Poésie Nègre et Malgache de Langue Française ${ }^{27}$ das Dilemma, in dem sich selbstbewusste Bewohner*innen Afrikas und der Antillen bis heute befinden: Sie bleiben auf die Sprachen der früheren Kolonisatoren und die ästhetischen Normen Europas verwiesen, so sie auf globalen Plattformen gesehen und gehört werden wollen. Den Zwang zur Einfügung in kulturfremde Ausdrucksmuster charakterisiert Sartre als äußerst gewaltsamen Vorgang: als Eindruck der afrikanischen und antillanischen Autoren, ein westlicher Geist stehle ihnen die Ideen, die Weißen Wörter schlürften ihr Denken auf. Ja, die Struktur eines Ausdrucksmediums übe so viel Zwang aus, dass innen das Artikulationsvermögen überhaupt verloren gehe. Auf diese sprachlich-kulturelle Spaltung werden in der Ethnologie denn auch bis heute gewisse Vorgänge von Geistbesessenheit zurückgeführt und

25 James Clifford, RETURNS. Becoming Indigenous in the Twenty-First Century, Cambridge/London: Harvard Univ. Press, 2013, 24.

26 James Clifford, RETURNS. Becoming Indigenous in the Twenty-First Century, Cambridge/London: Harvard Univ. Press, 2013, 25.

27 Leopold Sédar Senghor. Anthologie de la Nouvelle Poésie Nègre et Malgache de Langue Française, Paris: PUF, 1969 [1948]. $\mathrm{zu}$ "possession [...] staging of the foreigness of language" 28 erklärt.

Die in den französisch verfassten Gedichten der afrikanischen und antillanischen Autoren vorgebrachte Forderung nach Abbau der WeiBen Mauern wirft heute, knapp 70 Jahre später, die Frage auf, inwiefern die westlichen Normen noch immer Ausgangspunkt des Nachdenkens über nicht-westliche/-nördliche Kulturproduktionen sind. Wenn Sartre die Hoffnung formuliert, die Schwarzen möchten in ihre psychische Tiefe hinabsteigen, um das europäische Erbe in sich abzubauen und im eigenen Sprechen zu kollektiver Poesie zu gelangen, so nimmt er Edouard Glissants, des martinikanischen Poeten und Theoretikers, späteres Kulturprogramm vorweg. Denn Glissant erblickt in den 1980er Jahren die Möglichkeit des Abbaus des europäischen Erbes seinerseits darin, ${ }^{29}$ in das je besondere kulturelle Dunkel hinabzusteigen und den Aufstieg ans Licht der Aufklärung zu verweigern. Der westlich universalisierten Forderung nach Transparenz hält er ein Recht auf "Opazität", ein Recht auf kulturelle Undurchdringlichkeit entgegen. In der von seinem Landsmann Aimé Césaire mitbegründeten Négritude-Bewegung erblickt er zwar eine berechtigte Antwort auf den Wunsch, eine gemeinsame Herkunft und ein psychisches Gleichgewicht in der Vorstellung "eines" Volkes wiederzufinden; er kritisiert sie gleichwohl als zu afrikazentrierte Bewegung, die sich zudem in der Kolonisatorensprache vermittelt, und nennt sie ein überholtes Konzept. Die Idee der "retour en Afrique" müsse, so Glissant, in eine Taktik der "détour" umgebogen werden, der Schrei müsse sich zu chaotischer Vielstimmigkeit multiplizieren.

Er setzt ihr daher den Begriff der "Antillanité" als Ausdruck der Solidarität einer als dezidiert multiethnisch und -lingual verstandenen Region entgegen, für die „Beziehungen" konstitutiv seien, weshalb er eine Poétique de la rela-

28 Rosalind C. Morris, "Sprit Mediumship in the Age of New Media". In: Heike Behrend/Anja Dreschke/Martin Zillinger (Hgg.), Trans Mediums \& New Media. Spirit Possession in the Age of Technical Reproduction, New York: Fordham Univ. Press, 2015, 25-55 (40).

29 Edouard Glissant, Le Discours Antillais, Paris, 1981; Zersplitterte Welten. Der Diskurs der Antillen, Heidelberg, 1986. 
$\operatorname{tion}^{30}$ entwirft. Auf diese „Poetik der Relation" antworten wiederum die karibischen Schriftsteller Raphael Confiant und Patrick Chamoiseau mit dem Begriff der "Créolité", der seinerseits eine subversive Verwendung, semantische Anreicherung und Gegenrhythmisierung des Französischen wiedergeben und gerade nicht auf die Antillen beschränkt verstanden werden soll. Und noch einmal respondiert Glissant mit einem modifizierten Begriff, jenem der "Créolisation", da ihm Créolité trotz aller anders gelagerten Programmatik zu starr und homogenisierend erscheint. Kreolisierung der französischen Sprache - das ist für inn jenes gewünschte umwegige und unabschließbare Verfahren im Sinne der unumgänglichen kompositkulturellen Identitätsbildung auf der Basis indigener, afrikanischer und europäischer Kulturschichten und ihrer schrägen Verknüpfung. Verwurzelung und Entwurzelung greifen hier ineinander, auch wenn das Komposit von Insel zu Insel nach Maßgabe der kolonialbedingten Wiederholungs- und Differenzierungsweisen variiert. Glissants ästhetisch-politisches Modell des karibischen Archipels skizziert einen diskontinuierlichen Raum transversaler Bezugnahmen, wechselseitiger Durchdringung und je anderer ästhetischer Zusammensetzung, in der die einzelnen Elemente gleichberechtigt nebeneinander bestehen und das Nicht-Vermittelbare performieren. In Vorwegnahme von Mbembes Konzept einer generalisierten "Schwarzen Vernunft" schreibt Glissant der Kreolisierung ein "Ganze-Welt-Werden"31 zu, da sie die ganze Welt, rhizomatisch verflochten, in fortwährende Erschütterung versetze, in simultane und (nicht-in)dividuelle Verteilung und Neuaufteilung kultureller Differenz.

\section{Zeitgenössische Dividuationen}

Künstlerischen Verfahren, die die sprachlich-ästhetischen Spaltungen produktiv zu machen, die westlich/nördlichen Normen zu konterkarieren und innen wie im karibischen Kreolisch die ,Weißheit' auszutreiben suchen, begegnet man in der afrikanischen Kunst der Gegenwart zuhauf.

30 Edouard Glissant, Poétique de la relation, Paris: Gallimard, 1990.

31 Glissant, Traité du Tout-Monde, Paris: Gallimard, 1997.
Gemäß einer weiteren Devise von Achille Mbembe sucht die zeitgenössische "afropolitane" Kunst sich als avanciertes Modell zu verstehen, das sich in einem affirmierten "Zwischen" der Kulturen positioniert. Wie zeitgenössische weltläufige Personen, aus Afrika' sei diese Kunst durch ein "Ineinandergreifen von Hier und Woanders", eine "Präsenz des Woanders im Hier", wie er in seiner jüngst auf Deutsch erschienenen Schrift Ausgang aus der langen Nacht ${ }^{32}$ sagt, charakterisiert. Ergänzend zur Kritik der Schwarzen Vernunft macht er nun deutlich, dass es in der afropolitanen Ästhetik um ein dem herkömmlichen Stereotyp entgegengesetztes Afrikabild geht: um die Darstellung eines Selbstverständnisses von Afrikaner*innen als handlungsmächtigen, selbstreflexiven, affirmativ kompositkulturellen Identitäten, die ihr besonderes In-der-Welt-Sein und ihre kulturell-politische Differenz in selbstgewählte Partizipations- und Artikulationsweisen übersetzen.

Auf verwandte Weise akzentuiert der Kurator Okwui Enwezor ${ }^{33}$ die afrikanischen Kunstpraktiken von heute als vielfältige Aneignungs- und Modifikationsverfahren: Seien sie doch durch unterschiedliche Rückgriffe auf die Tradition und durch unterschiedliche Rekombinationen der globalisierten Kunstsprachen charakterisiert. Ihre Heterogenität bringt er in deutlicher Nähe zum karibischen Diskurs auf die Formel: "a series of shifting grounds composed of fragments, of composite identities, and micro-narratives." ${ }^{34}$ Schon mit Blick auf den verwertungshungrigen Kunstmarkt warnt er davor, sich auf eine ethnische oder geographische Herkunft festlegen zu lassen und rät stattdessen an, eine post-ethnische Identität zu entwickeln und eine Kunst des Zitats und des Supplements zwischen unterschiedlichen Archiven und Traditionen, zwischen diasporischen und kosmopolitischen Räumen zu praktizieren. ${ }^{35}$ Der

32 Achille Mbembe, Ausgang aus der langen Nacht. Versuch über ein entkolonisiertes Afrika, Berlin, 2016, 285.

33 Okwui Enwezor. "Situating Contemporary African Art: Introduction". In: ders. u. a. (Hgg.), Contemporary African Art since 1980. Bologna: Damiani, 2009, 9-50.

34 Okwui Enwezor. "Situating Contemporary African Art: Introduction". In: ders. u. a. (Hgg.), Contemporary African Art since 1980. Bologna: Damiani, 2009, 11.

35 Okwui Enwezor. "Situating Contemporary African Art: Introduction". In: ders. u. a. (Hgg.), Contemporary African Art since 1980. Bologna: Damiani, 2009, 26. 
Philosoph Paul Hountondji aus dem Benin fordert seinerseits im Sinne artikulatorischer Vielfalt, aber auch zeitgemäßer Personenverständnisse, den Mythos der Afrikanität zu dekonstruieren und sich von ethnozentrischen Denkansätzen zu verabschieden: „it was necessary to begin by demythifying the concept of Africanity. (...) In order to think of the richness of African tradition, it was necessary to weaken resolutely the concept of Africa, to rid it of all its ethical, religious, philosophical, political connotations" 36 .

Der kamerunische Filmemacher Jean-Pierre Bekolo wählt daher für seinen Experimentalfilm Naked Reality (2016) eine poetisch-assoziative Ausdrucksform, die sich wie bereits sein Spielfilm Aristotle's Plot (1995) den europäisch normierten Filmdramaturgien zu entziehen und einen kulturübergreifenden Echoraum zu eröffnen sucht. Zur afropolitanen Äußerungsweise von Aristotle's Plot gehört, dass er die Bedingtheit des afrikanischen Films durch das europäisch-dramaturgische Format ebenso problematisiert wie die freiwillige Orientierung des afrikanischen Kinopublikums an US-amerikanischen Actionfilmen und ihren Heldenfiguren, ihre mangelnde Suche nach alternativen Ausdrucksweisen wie ihr fehlendes Engagement für die Filmkunst überhaupt. Der Film demonstriert nicht nur, dass die von Aristoteles geforderte lineare Handlungsführung unter afrikanischen Verhältnissen nicht einlösbar ist, sondern auch, dass die klischeehaften Bilder des afrikanischen Kontinents die aristotelische Poetik mehr als gewünscht beglaubigen, indem sie beim Kinopublikum fortgesetzt die Affekte von Mitleid und Schrecken hervorrufen. Die Hauptleistung von Bekolos Film besteht aber darin, nicht die europäische und afrikanische Welt einander gegenüberzustellen, sondern ihre kulturelle Verwiesenheit und medienbedingte Dividuation vorzuführen, unterschiedliche filmästhetische Haltungen miteinander zu konfrontieren, dabei für ein afropolitan-experimentelles Kino zu werben und einen Raum ästhetischer Unbestimmtheit zu eröffnen.

Erkenntnisträchtiger als europäisch renationalisierte Kulturstatements erscheinen daher zeitgenössische Kunst- und Theorieproduktionen aus der nicht-westlich/-nördlichen Welt. Im Austrag

36 Paul Hountondji. „Que peut la philosophie?" In: Présence Africaine, no. 119. Dakar/Paris, 1981, 52. ästhetischer Differenzen und in ihrer selbstreflexiven Kritik sind sie wegweisend für ein mondialisiertes In-der-Welt-Sein und eine kompositkulturelle personale Beweglichkeit. Allgemein lässt sich beobachten, dass der afrikanische und karibische Diskurs auf den Export europäischer Wissensmodelle und ästhetischer Normen heute weniger mit Ablehnung als mit dem Appell reagiert, diese kritisch zu adaptieren, gegebenenfalls parodistisch umzukehren und mit Elementen aus vielfältigen Quellen zu mischen, auf dass zuletzt keine eindeutige Herkunft der kulturellen Artikulation mehr ausmachbar ist. Anspruchsvolle Diskurse und Kunstproduktionen aus dem globalen Süden stellen heute ihre notwendige Verwiesenheit auf diverse "Andere" und ihre dividuelle Verfasstheit aus; sie suchen sich lose und dennoch spezifisch mit ausgewählten Bild- und Tonarchiven zu verbinden und sich darüber ethnischen Festlegungen zu entwinden.

Rew Chow geht so weit zu behaupten, dass die Annahme eines - westlichen - Originals und einer davon abweichenden "Andersheit" heute sowieso unmöglich geworden ist, da jede symbolische Produktion - wie etwa der Film - durch zahlreiche mediale Übersetzungsprozesse gegangen ist und daher bereits als intermedial und interkulturell zu bezeichnen ist:

Precisely because of the deadlock of the more or less complete Europeanization of the world, which has led not only to an organization of these cultures by way of European languages, philosophies, and sciences, the recourse to the archaic, authentic past of other cultures (...) is a futile one. Critiquing the great disparity between Europa and the rest of the world means not simply a restitution of the origin that is Europe's other but a thorough dismantling of both the notion of origin and the notion of alterity as we know them today. ${ }^{37}$

Sie plädiert ihrerseits für das Zugeständnis einer "coevalness of cultures" und fordert im Hinblick darauf einen "dritten Term" der Übersetzung, der dem Dualismus von Original und Übersetzung ausweicht, aber auch die (Re)Produktion (post) kolonialer Asymmetrien innerhalb der zeitgenössischen kulturellen Komposite deutlich macht:

Besides acknowledging the co-temporality of cultures through our media, the 'third term' would also mean

37 Rey Chow, Primitive Passions, New York: Columbia Univ. Press, 1995, 194. 
acknowledging that the West's 'primitive others' are equally caught up in the generalized atmosphere of unequal power distribution and are actively (re)producing within themselves the structures of domination. ${ }^{38}$

Nur im Diesseits der Gegensätze von Europa und dem "Anderen", von Original und Übersetzung können sich die zwangsweise "Veränderten" als ebenfalls kompositkulturelle Teilhaber*innen erkennen, die die erfahrenen Dominanzverhältnisse nun unter Umständen in sich selbst reproduzieren. Erst ein dritter Ort würde erlauben, den westlich/nördlichen Anthropologien und Ethnographien ihre visualistische epistemologische Basis zu entziehen und die unhintergehbaren kulturellen Dividuationen zu analysieren.

Im Hinblick auf die Konzeptualisierung dieser intrikaten Verhältnisse taucht auch der von LévyBruhl geprägte Begriff der Partizipation im kulturwissenschaftlichen Diskurs wieder auf. Die von ihm betonte "Konsubstantialität des Eigenen und Anderen"39 in der "primitiven" Kultur erscheint nun als adäquate Bestimmung personal-kultureller Verfasstheiten der Gegenwart. Und auch der von Marilyn Strathern ${ }^{40}$ geprägte Begriff des "Dividuellen", mit dem sie nicht-dualistische Verhältnisse zwischen den Einzelnen und dem Kollektiv in melanesischen Geschenkökonomien wiedergibt, erscheint heute geeignet zur Akzentuierung der vieldirektional-medialen Einlassungen von Personen, welche ihre Zugehörigkeit zu gewissen Kollektiven damit einerseits begründen, andererseits verunschärfen. Der Begriff der Dividuation steht aber noch für mehr: zum einen für die allgemeine Erkenntnis, dass menschliche Subjektivierungen heute konstitutiv mit nicht-zählbar vielen "Anderen" auf verschiedenen Ebenen verflochten sind, weshalb sie längst nicht mehr als Ungeteilte zu bezeichnen sind; zum anderen für die kulturbezogene Beobachtung, dass die Zeitgenossen in der Regel an mehreren Sprachen, gesellschaftlichen Normen und symbolischen Systemen zugleich partizipieren und damit selbst kulturelle Komposite geworden sind. Aus diesem Grund sind sie aufgerufen, ihre jeweils besondere

38 Rey Chow, Primitive Passions, New York: Columbia Univ. Press, 1995, 195.

39 Vgl. Lucien Lévy Bruhl, Das Denken der Naturvölker, Wien/Leipzig: Braumüller, 1921, 57-58.

$40 \mathrm{Vgl}$. Marilyn Strathern. The Gender of Gift. Los Angeles: Univ. of California Press, 1988, 13. psychophysische und kulturelle Komposition, die mit "Anderen" geteilten Schnittmengen, aber auch ihre unfreiwilligen (Selbst-)Unterteilungen, ihre nur bedingte Kohärenz im Sinne eines metastabilen Gleichgewichts zu moderieren und so weit als möglich ihre Teilhabezwänge zu unterbrechen. Dasselbe gilt für nationalstaatlich oder territorial definierte Kulturen, die ihre dividuelle Verfasstheit, das Ineinander kulturspezifischer Ensembles im Sinne vermehrter Inklusion und der Vermeidung von Gewalt als für die Gegenwart erkenntnisträchtige und zukunftsweisende Modi anerkennen sollten.

\section{Literaturverzeichnis}

Behrend, Heike/Dreschke, Anja/Zillinger, Martin

(Hgg.) (2015): Trans Mediums \& New Media. Spirit Possession in the Age of Technical Reproduction. New York: Fordham Univ. Press.

Bergson, Henri (1889): Essai sur les données immédiates de la conscience. Paris: Presses univ. de France.

Buden, Boris/Nowotny, Stefan (Hgg.) (2008): Übersetzung: Das Versprechen eines Begriffs. Wien: Turia + Kant.

Buden, Boris (2008): Doris Bachmann-Medick im Gespräch mit Boris Buden. In: Buden, Boris/Nowotny, Stefan (Hgg.): Übersetzung: Das Versprechen eines Begriffs. Wien: Turia + Kant, S. 29-42.

Chow, Rey (1995): Primitive Passions. Visuality, Sexuality, Ethnography, and Contemporary Chinese Cinema. New York: Columbia Univ. Press.

Clifford, James (2013): RETURNS. Becoming Indigenous in the Twenty-First Century. Cambridge/London: Harvard Univ. Press.

Conrad, Sebastian et al. (Hgg.) (2002): Jenseits des Eurozentrismus. Postkoloniale Perspektiven in den Geschichts- und Kulturwissenschaften, Frankfurt a.M./New York: Campus Verlag.

Deleuze, Gilles (1993): Postscriptum zur Kontrollgesellschaft, in: Unterhandlungen, Frankfurt/M.: Suhrkamp.

Deleuze, Gilles (1996): Das Bewegungs-Bild. Kino 1. Frankfurt/M.: Suhrkamp.

Diagne, Souleymane Bachir (2010): Bergson postcolonial. Paris: CNRS Ed.

Enwezor, Okwui (2009): Situating Contemporary African Art: Introduction. In: ders. et al. (Hgg.), Contemporary African Art since 1980. Bologna: Damiani, S. 9-50.

Enwezor, Okwui et al (2009): Contemporary African Art since 1980. Bologna: Damiani.

Frobenius, Leo (1933): Kulturgeschichte Afrikas. Prolegomena zu einer historischen Gestaltlehre. Frankfurt/M.: Phaidon.

Gilroy, Paul (1993): The Black Atlantic. Modernity and Double Consciousness. London/New York: Verso. 
Glissant, Edouard (1981): Le Discours Antillais. Paris: Seuil.

Glissant, Edouard (1986): Zersplitterte Welten. Der Diskurs der Antillen. Heidelberg: Wunderhorn.

Glissant, Eduoard (1997): Traité du Tout-Monde, Paris: Gallimard.

Hall, Stuart (2002): Wann gab es, das Postkoloniale'? Denken an der Grenze, in: Sebastian Conrad et al. (Hgg.), Jenseits des Eurozentrismus. Postkoloniale Perspektiven in den Geschichts- und Kulturwissenschaften, Frankfurt/M./New York: Campus Verlag, 197-223 (212).

Hountondji, Paul (1981): Que peut la philosophie?. In: Présence Africaine, no. 119, S. 47-71.

Lévy-Bruhl, Lucien (1910): Les fonctions mentales dans les sociétés inférieures. Paris: Alcan.

Lévy-Bruhl, Lucien (1921): Das Denken der Naturvölker, Wien/Leipzig: Braumüller.

Lévy-Bruhl, Lucien (1922): La mentalité primitive. Paris: Presses univ. de France.

Lévi-Strauss, Claude (1962): La pensée sauvage, Paris: Plon.

Mbembe, Achille (2013): Critique de la raison nègre, Paris: La Découverte.

Mbembe, Achille (2014): Kritik der Schwarzen Vernunft, Berlin: Suhrkamp.

Mbembe, Achille (2016): Ausgang aus der langen Nacht. Versuch über ein entkolonisiertes Afrika. Berlin: Suhrkamp.

Morris, Rosalind C. (2015): Sprit Mediumship in the Age of New Media. In: Behrend, Heike/Dreschke, Anja/
Zillinger, Martin (Hgg.): Trans Mediums \& New Media. Spirit Possession in the Age of Technical Reproduction. New York: Fordham Univ. Press, S. 25-55.

Ott, Michaela (2015): Dividuationen. Theorien der Teilhabe. Berlin: b_books.

Senghor, Leopold Sédar (1967): Négritude und Humanismus, übers. v. Jan Heinz Jahn. Düsseldorf: Diederichs.

Senghor, Leopold Sédar (1968): Négritude et Germanisme. Tübingen/Basel: Horst Erdmann.

Senghor, Leopold Sédar (1969 [1948]): Anthologie de la Nouvelle Poésie Nègre et Malgache de Langue Française. Paris: PUF.

Stoler, Ann Laura (1995): Race and the Education of Desire: Foucault's ,History of Sexuality' and the Colonial Order of Things, Durham/London: Duke University Press.

Spivak, Gayatri Chakravorty (1999): A Critique of Postcolonial Reason. Toward a History of the Vanishing Past, Cambridge (Mass)/London: Harvard Univ. Press.

Strathern, Marilyn (1988): The Gender of Gift. Los Angeles: Univ. of California Press. 